\title{
INFRARED ANALYSIS OF SUPERNOVA REMNANTS
}

\author{
Eli Dwek \\ Laboratory for Astronomy and Solar Physics \\ NASA/Goddard Space Flight Center, Maryland
}

\begin{abstract}
Infrared observations of supernova remnants obtained with the Infrared Astronomical Satellite provide new insights into the dynamics and energetics of the remnants, and into their interaction with the ambient interstellar medium. In most remnants the infrared emission arises from dust that is collisionally heated by the $\mathrm{X}$-ray emitting gas. The infrared observations can therefore be used as a diagnostic for the physical conditions of the shocked gas. In particular, it is shown that all the prominent $\mathrm{X}$-ray remnants in the Galaxy and in the LMC cool mainly by dust grain collisions instead of atomic processes.
\end{abstract}

\section{Introduction}

Theoretical calculations (Ostriker and Silk 1973; Silk and Burke 1974) suggest that at temperatures above $\sim 10^{6} \mathrm{~K}$, a dusty plasma should cool mainly at infrared (IR) wavelengths. Supernova remnants (SNR) should therefore appear as prominent IR sources in the galaxy. However, searches for IR emission from Tycho, Kepler, and Cas A (Wright et al. 1980), and the Crab Nebula (Harvey et al. 1978), yielded essentially negative results. Searches for IR emission from the optical knots in Cas A, which were suggested by Dwek and Werner (1981) as possible sites of supernova condensate material, were more successful (Dinerstein et al. 1987). However, the detected radiation has been attributed to IR line emission from ionic species present in the shocked optical nebulosities of Cas A.

This situation has drastically changed with the success of the Infrared Astronomical Satellite (IRAS) which in November 1983 completed an unbiased all-sky survey at 12, 25, 60, and $100 \mu \mathrm{m}$. Details on the instrument, observing strategy, and mission objectives can be found in Neugebauer et al. (1984), the first paper in a special Ap. J. Letters issue dedicated to the IRAS mission. In the same issue, Marsden etal. (1984) reported the first detection of IR emission from dust in a SNR, the Crab Nebula. Figure 1, taken from Mezger et al. (1986), shows the spectrum of the IR and radio emission from the Crab. The Crab has a strong non-thermal radiation field that fills the nebula, and the figure clearly shows the thermal IR emission just barely rearing its peak above this nebular emission component. Dwek and Werner (1981) have shown that in the Crab the dust is most likely to be radiatively heated by the ambient radiation field. The Crab nebula may therefore represent a special class of SNR in which the IR emission represents only a minor fraction of the total radiative output from the remnant.

The opposite is true of the emission of all other remnants that will be discussed in this review. Figure 2 (based on Dwek et al. 1987a) depicts the power emitted by Cas A from radio to X-ray wavelengths. The figure shows that most of the radiative output of the remnant is at IR wavelengths. The dust is most likely of interstellar origin and collisionally heated by the shocked gas. 


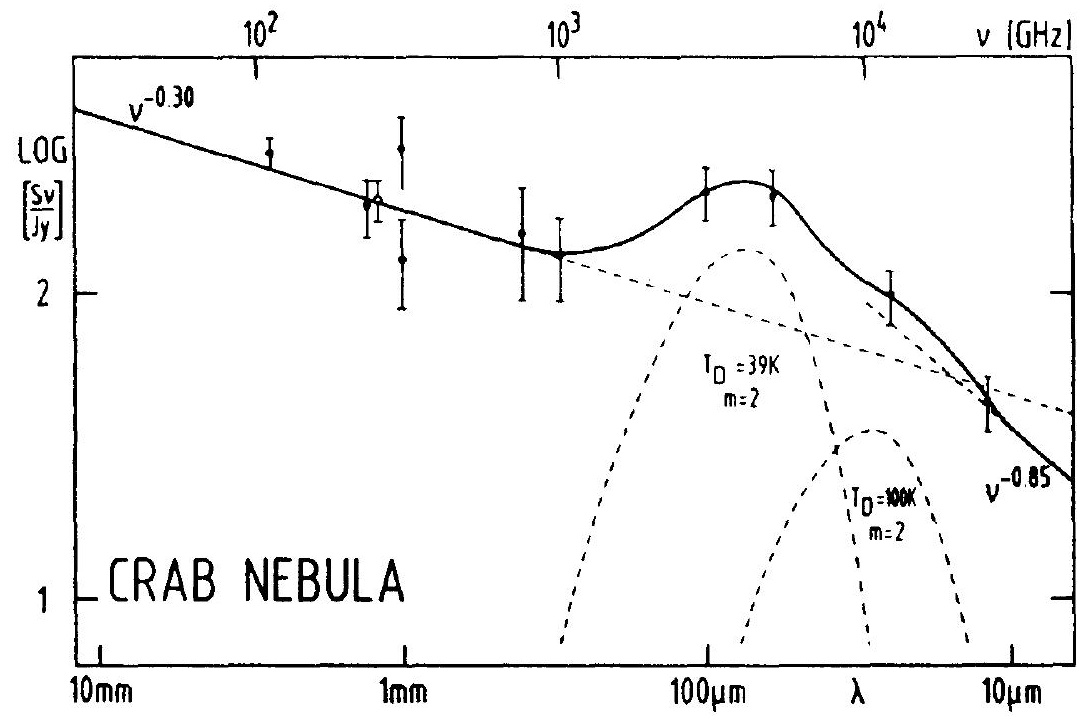

Figure 1 - The spectrum of the Crab Nebula as presented by Mezger et al. 1986. The dust is radiatively-heated in the Crab, and its IR emission represents only a small fraction of the total radiative output from the nebula.

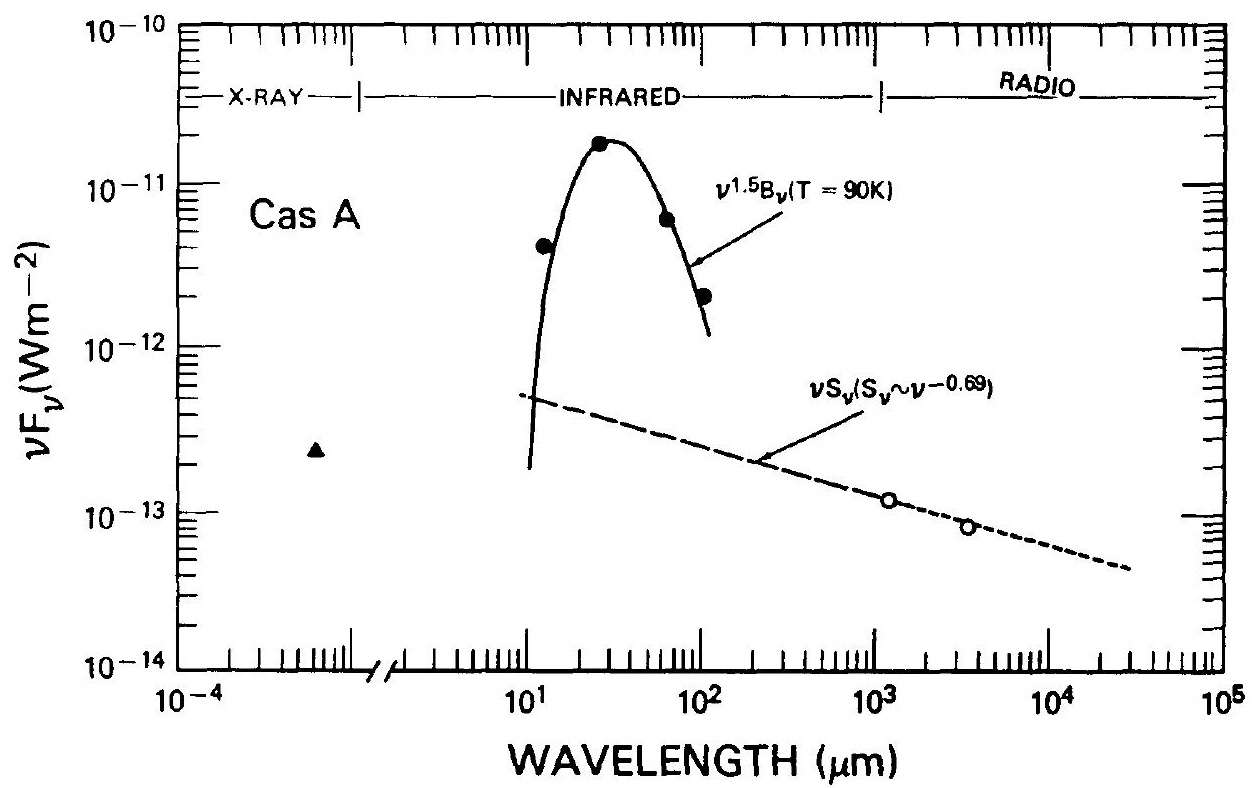

Figure 2 - The power spectrum of Cas A. The dust is collisionally-heated in the remnant, and its IR radiation dominates the emission at all other wavelengths.

The most extensive search for SNR in the IRAS skyflux plates is being conducted by Arendt (1987a). From a preliminary unbiased sample of 70 remnants, identified by their radio emission, 16 showed evidence for IR emission that is clearly associated with the remnant, and 24 sources showed evidence for IR emission, but its association with the remnants is uncertain. The remaining 30 regions showed no evidence for IR emission that could be attributed to the remnant. This investigation shows that the IR detection of SNR is mostly hampered by confusion with other IR 
objects near the galactic plane. The latter is suggested by preliminary findings that the average galactic lattitude of the remnants detected in the search is $|b|=2.0^{\circ}$, whereas remnants with no detectable IR emission seem to be located closer to the plane with an average value of $|b|=0.8^{\circ}$. However, some remnants elude detection even though they are high-lattitude objects, and are therefore expected to figure quite prominently in the IRAS skyflux plates. An example of such a remnant is SN $1006\left(l ; b=327.5^{\circ} ;+14.6^{\circ}\right)$ which is expanding into a low-density interstellar medium (ISM). This suggests that a minimum ISM density is required to observe the IR emission from shock heated dust (Dwek et al . 1987b; Braun 1986a).

In the remainder of this review I will emphasize remnants that are most prominent in the IR. Multiwavelengths analysis of these remnants have been presented in a series of papers: Tycho, Kepler, and Cas A (Braun 1987), Cas A (Dwek etal. 1987a); IC443 (Braun 1986b; Mufson et al. 1986; McCollough and Mufson 1987); Cygnus Loop (Braun 1986c); and G292.0+1.8 (Braun 1986a). For these remnants, the IRAS observations constitute the first observational evidence of collisionally heated dust in the ISM. The IR emission may therefore be used as a diagnostic for the plasma conditions in the remnant , and a new means for deriving various parameters that determine its evolution.

\section{Infrared Emission Mechanism}

The galaxy is transparent at wavelengths of the IRAS bands. Therefore, several dust components not associated with the remnant may contribute to the IR flux from the direction of the remnant. These include: 1) emission from interplanetary dust particles (zodiacal dust); 2) emission from interstellar dust; 3) infrared cirrus; and 4) any galactic or extragalactic extended or point sources. Any attempt to analyze the $\mathbb{R}$ emission from the remnant must therefore be preceded by a separation of all these various emission components.

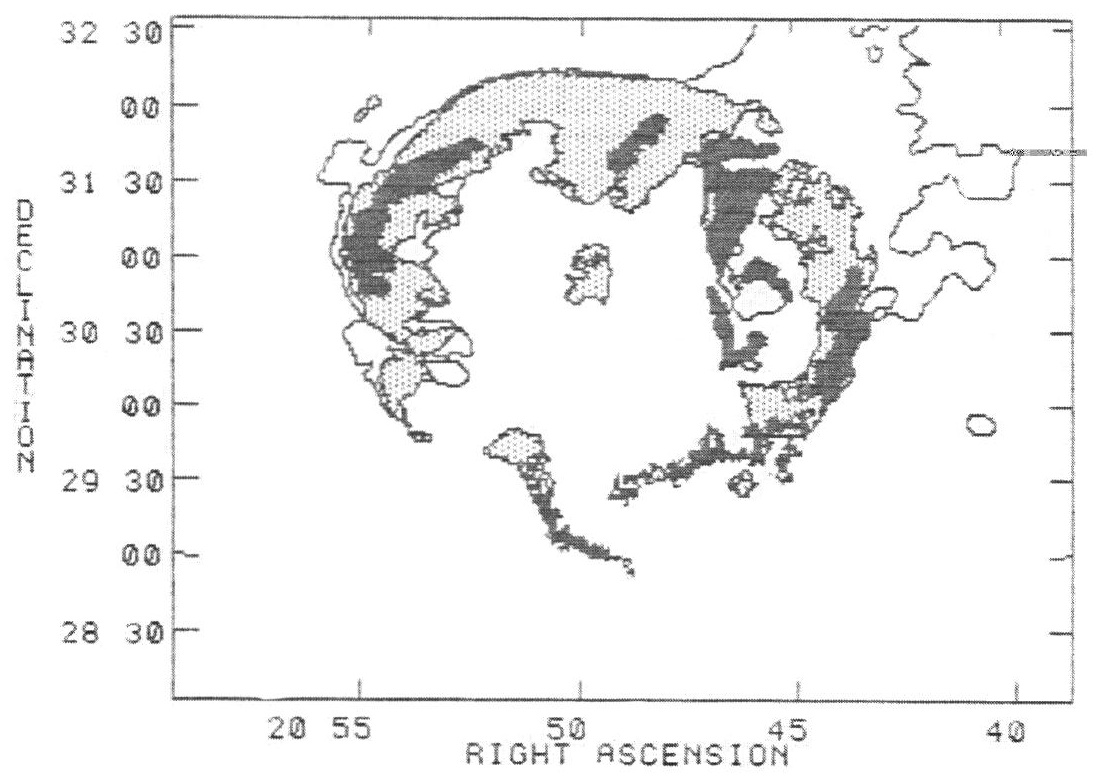

Figure 3 -A composite image of the Cygnus Loop at $60 \mu \mathrm{m}$ (outer contour lines), $X$-ray (light shaded area), and optical wavelengths (dark shaded areas).

The main zodiacal emission component (described by Hauser et al, 1984) has a flat spatial 
distribution over the dimensions of all extended remnants, provided they are sufficiently far removed from the ecliptic plane. The contribution of this component to the IR can therefore be removed from the data by the subtraction of a flat or smoothly varying background. The same is true for the interstellar dust component whose emission, a few degrees from the galactic plane, can be described by a cosec law in galactic latitude. The infrared cirrus, a new component of IR emission discovered by the IRAS (Low et al, 1984), consists of dust heated by the interstellar radiation field, and exhibits filamentary structure on all scales. However, since its morphology and color temperature are significantly different from that of the shock-heated dust, its contribution can be separated from that of the remnant by decomposing the emission into its various spectral components. Finally, extended IR sources are easily distinguishable and separable from the remnant emission. A good example of a region confused by zodiacal light, cirrus, galactic emission , and IR point sources, where the IR emission was separated into its various spectral components, is the Cygnus Loop (Braun 1986c). Figure 3 is a composite image of the Cygnus Loop at $60 \mu \mathrm{m}$, $\mathrm{X}$-ray, and optical wavelengths. The figure shows that the IR emission from dust arises from the same region that gives rise to the $\mathrm{X}$-ray emission, except for regions where the IR emission is dominated by cirrus, and regions where the $\mathrm{X}$-ray is obscured by foreground material (the 'carrot' is such a region). Enhanced IR emission arises from the shocked, optical line emitting filaments.

Figure 4 is a schematic diagram of a supernova remnant expanding into a cloudy ISM depicting various sites and sources of infrared emission. The expanding SN blast wave sweeps up interstellar dust (or circumstellar dust in young remnants) that is collisionally heated by the X-ray emitting gas. The blast wave may engulf a nebulosity or impinge upon a molecular cloud. Infrared lines from atomic or ionized species, continuum free-free emission, and thermal emission from dust are the main sources of IR emission expected from these nebulosities. Table 1 lists several lines that may be important in the IRAS bands. In young remnants, the pressure behind the forwardly expanding blast wave will drive a reverse shock through the cooling ejecta. If dust formed in this metal-rich material, this shock will radiate mostly at IR wavelengths. Finally, in centrally-filled, Crab-like remnants, the dust may be heated by the ambient non-thermal radiation field.The wide variety of IR emission mechanisms and sites discussed above demonstrates the usefulness of using IRAS data as a diagnostic tool for studying the evolution and interaction of supernova remnants with the ISM.

\section{Table 1}

Infrared Lines in the IRAS Bands

\begin{tabular}{ll}
\hline IRAS Band & Atomic/Ionic Specie \\
\hline & \\
$12 \mu \mathrm{m}$ & S IV $(10.5 \mu \mathrm{m})$, Ne II $(12.8 \mu \mathrm{m})$ \\
$25 \mu \mathrm{m}$ & S III $(18.7 \mu \mathrm{m})$, Fe III $(22.9 \mu \mathrm{m})$, Fe II $(26.0 \mu \mathrm{m})$ \\
$60 \mu \mathrm{m}$ & O III $(51.8 \mu \mathrm{m})$, S I $(56.3 \mu \mathrm{m})$, O I $(63.2 \mu \mathrm{m})$, \\
& Si I $(68.5 \mu \mathrm{m})$ \\
$100 \mu \mathrm{m}$ & O III $(88.4 \mu \mathrm{m})$ \\
\hline
\end{tabular}

In most remnants (important exceptions to this statement will be discussed later on) the dominant source of IR emission is collisionally heated dust. Therefore, the study of the physical interaction between these dust particles and the hot gas can yield valuable information on plasma conditions and remnant parameters. 


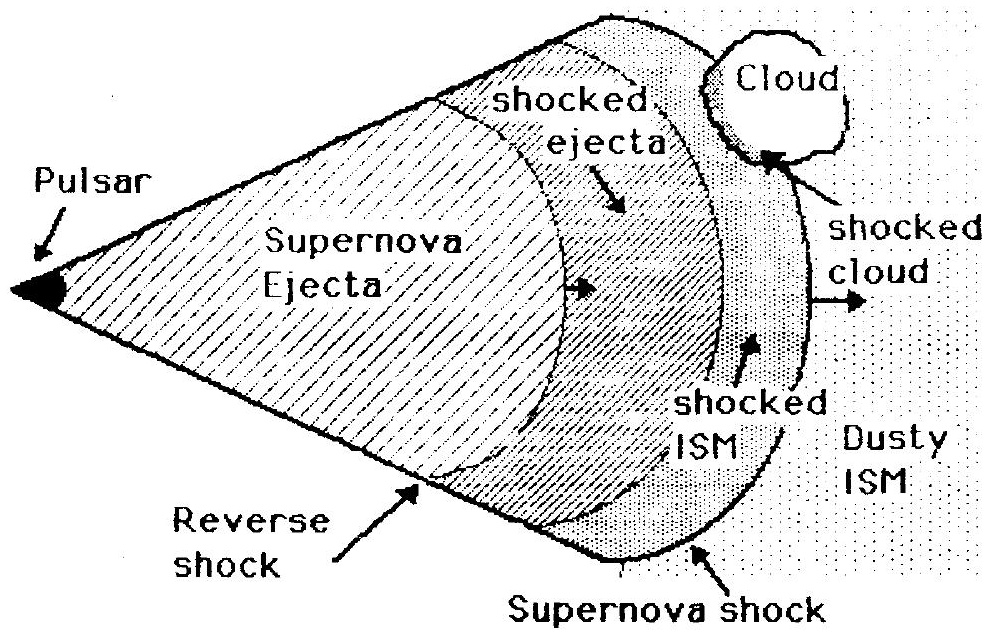

Figure 4 - A schematic diagram of a supernova remnant expanding into an inhomogeneous, dusty medium.

\section{The Physics of Dust Particles Behind Strong Shocks}

The IR emission from SNR is primarily determined by the initial conditions (i.e., the dust-to-gas mass ratio, and the grain composition and size distribution of the preshocked gas). After passing through the shock front, the dust is subjected to various physical processes (Burke and Silk 1974; Shull 1977; Draine and Salpeter 1979a, 1987b; Dwek 1987) that determine the efficiency at which the blast wave energy is converted to infrared emission. These include: 1) collisional charging of the dust; 2) grain destruction by sputtering or sublimation; and 3) collisional heating (mostly by electrons) of the dust grains.

The charge on the dust particle determines its effective collisional cross-section (Spitzer 1978), and how effectively it is coupled (via magnetic fields) to the shocked gas. In the absence of a magnetic field, a dust particle will move ballistically through the shock and spend a reduced fraction of its lifetime near the shock front. The calculations of Draine and Salpeter (1979a) show that at temperatures above $\sim 10^{6} \mathrm{~K}$, secondary electron emission becomes important. Consequently, the grain charge is too small to affect its collisional heating rate with the ambient gas, but sufficiently large to effectively couple it to the shocked gas with moderate values of the magnetic field (Shull 1977).

Grain destruction by sputtering will shift the weight of any initial grain size distribution to smaller grain sizes, reducing the effective grain area available for heating. For gas temperatures above $\sim 10^{6} \mathrm{~K}$, the dust lifetime against sputtering is approximately given by $t(\mathrm{yr})=10^{6}$ $\mathrm{a}(\mu \mathrm{m}) / \mathrm{n}\left(\mathrm{cm}^{-3}\right)$ (Draine and Salpeter 1979a; Seab 1986), so that $0.1 \mu \mathrm{m}$ dust particles will survive for about $10,000 \mathrm{yrs}$ in a $10 \mathrm{~cm}^{-3}$ gas. This is longer than the shock crossing time in all the bright $\mathrm{X}$-ray remnants considered here.

The dust particles are primarily heated by electronic collisions, thereby cooling the gas. Figure 
5 (taken from Dwek 1987) depicts $\Lambda_{d}(T)$, the cooling function (in erg cm $\mathrm{s}^{-1}$ ) of a dusty plasma containing a cosmic abundance of interstellar graphite and silicate grains (Mathis, Rumpl, and Nordsieck 1977; hereafter MRN) whose grain size distribution has been extended to very small sizes (solid line). Thin lines in the figure correspond to various single-size distributions of silicate-graphite grains. At temperatures below $\sim 2 \times 10^{7} \mathrm{~K}$ (the exact value depends on the grain size), all the electrons are stopped in the grain, and $\Lambda_{d}$ increases as $T^{3 / 2}$. At higher temperatures, the dust particles become transparent to the incident electrons, and at $\mathrm{T}>3 \times 10^{8} \mathrm{~K}, \Lambda_{\mathrm{d}}$ actually decreases with gas temperature. However, at these temperatures, the ionic contribution to $\Lambda_{d}$ is still rising with gas temperature and equals that of the electrons, thus maintaining an approximately constant value of $\Lambda_{\mathrm{d}} \approx 5 \times 10^{-21} \mathrm{erg} \mathrm{cm}^{3} \mathrm{~s}^{-1}$ at $\mathrm{T}>2 \times 10^{7} \mathrm{~K}$.

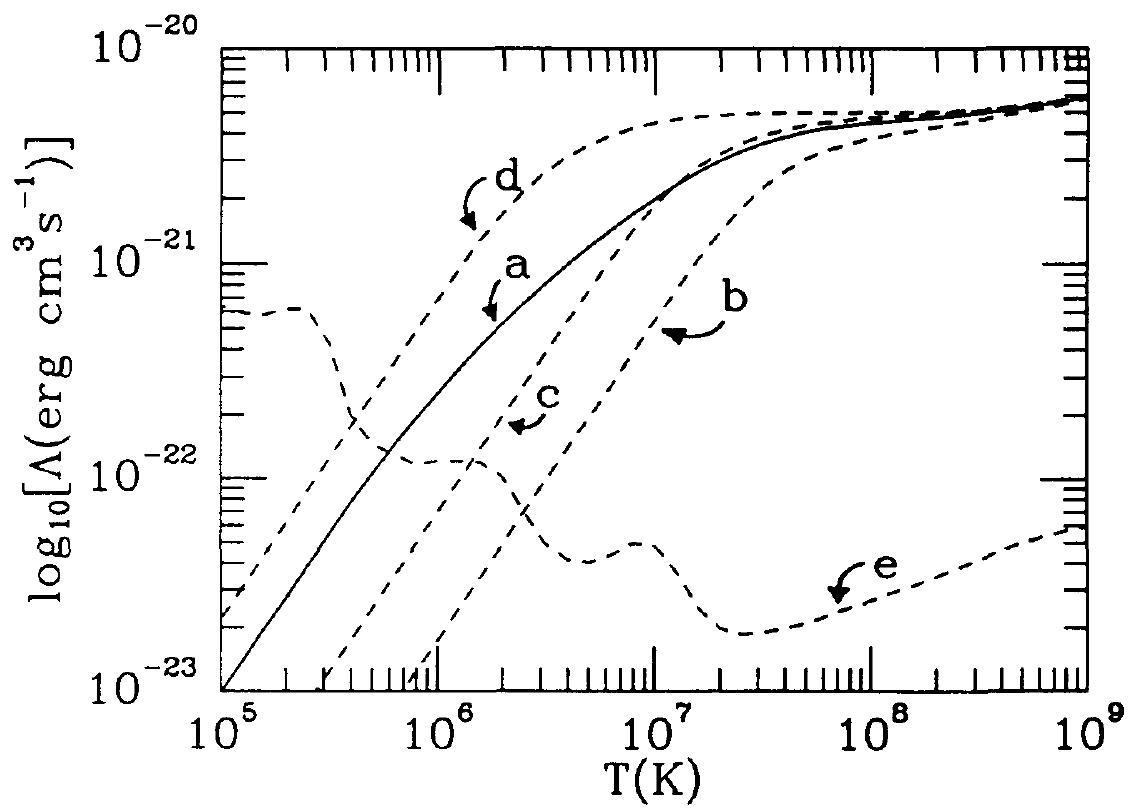

Figure 5 - The cooling function of a dusty plasma via gas-grain collisions (curve a) is plotted as a function of gas temperature for an MRN interstellar dust model in which the grain size distribution has been extended to very small grain sizes. Curve (e) is the cooling function of the gas due to atomic processes. For more information see Dwek (1987).

\section{The Infrared Cooling of A Dusty Plasma}

Also shown in the figure is $\Lambda(T)$, the cooling function of a plasma due to atomic (free-free, bound-free, and bound-bound) transitions (e.g. Raymond,Cox, and Smith 1976). The ratio between $\Lambda_{\mathrm{d}}$ and $\Lambda$ is a measure of the relative importance of the cooling mechanisms they represent. From the figure we see that plasma cooling by gas-grain collisions is the dominant cooling mechanism in the shocked gas, exceeding the atomic cooling rate by about two orders of magnitude at temperatures above $\sim 10^{7} \mathrm{~K}$.This fact may have a significant effect on the thermal and dynamical evolution of SNR. However, the dominance of IR cooling may not be realized in real astrophysical environments, and the ratio between the two cooling functions can deviate substantially from its theoretically predicted value. Reasons for these deviations may be: 1) the dust may be depleted 
compared with its average interstellar value in the shocked gas; 2 ) the dust may not be effectively coupled to the gas in the postshock region; 3 ) a significant fraction of the IR emission may originate from radiatively-heated dust that does not reside in the X-ray emitting gas; and 4) infrared lines may contribute significantly to the observed emission in the IRAS bands.

To compare the theory with the observations, it is useful to introduce an infrared-to-X-ray flux ratio (hereafter referred to as the $\mathrm{IRX}$ ratio) defined as

$$
\operatorname{IRX}=\Lambda_{\mathrm{d}}(\mathrm{T}) / \Lambda_{0.2-4.0^{(T)}}
$$

where $\Lambda_{0.2}-4.0(T)$ is the value of the atomic cooling function in the $0.2-4.0 \mathrm{keV}$ energy interval. For a given dust-to-gas mass ratio and dust model, and for a plasma that is in ionization equilibrium, the IRX ratio is only a function of gas temperature, which is graphically shown in Figure 6 (solid line). To construct an 'observed' IRX ratio, Dwek et al. (1987b) carried out a comparison between the IR and X-ray cooling rates of nine selected remnants including Cas A, Tycho, Kepler, SN 1006, RCW 103, IC 443, Puppis A, and the Cygnus Loop. Two corrections need to be made to the observed $X$-ray fluxes to allow for an intercomparison between remnants: 1) all flux estimates from the remnants must be converted to the chosen common X-ray band; 2) observed fluxes must be corrected for interstellar photoelectric absorption along the line of sight to the remnant; and 3) the observed flux must be corrected for non-equilibrium ionization effects. Hamilton, Sarazin, and Chevalier (1983) showed that a shocked plasma may not achieve ionization equilibrium, and its $\mathrm{X}$-ray emission will be significantly enhanced (by a factor $\eta$ ) compared to that expected from a remnant in ionization equilibrium. Therefore, for comparison with the theoretical curve (which assumes ionization equilibrium), the observed IRX ratio has to be increased by the factor $\eta$.

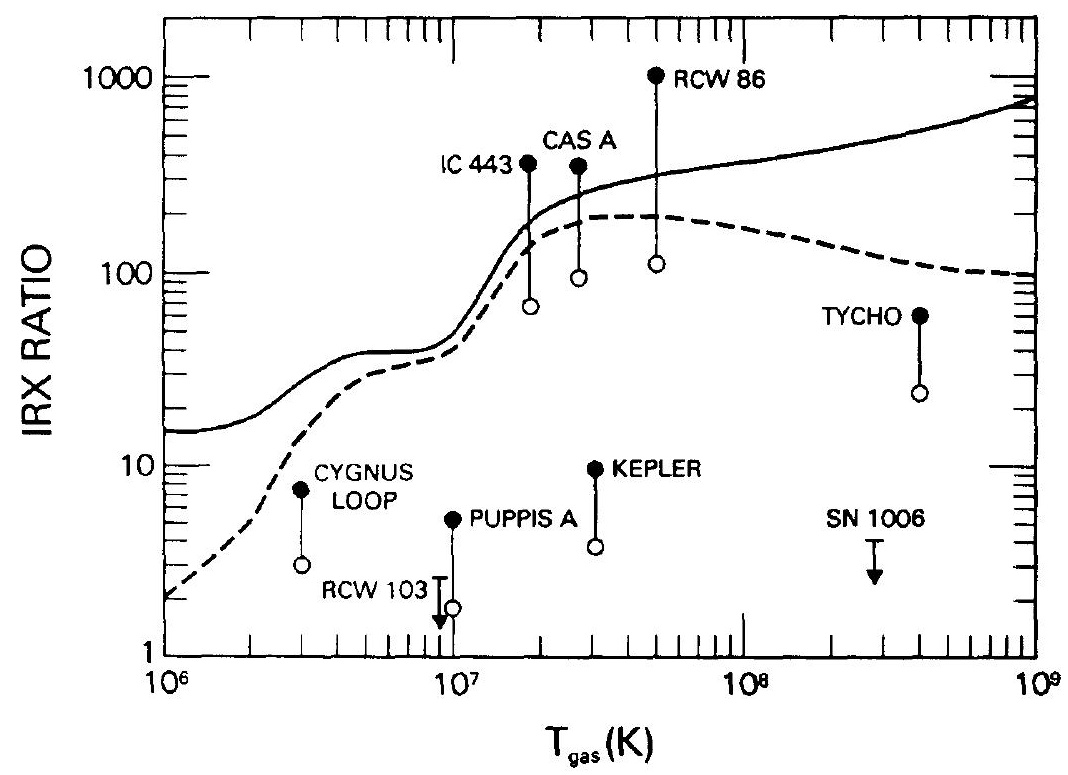

Figure 6 - The IRX ratio (defined by equation 1) is plotted here (solid line) as a function of gas temperature. Open circles represent the observed IR to $(0.2-4) \mathrm{keV}$ flux ratio of various $S N R$, and the filled circles represent these flux ratios, corrected for non-equilibrium ionization effects in the remnant. 
Figure 6 shows the result of the comparison. The IRX ratio, as defined in eq. (1), is plotted as a solid line in the figure. The dashed line represents the ratio of the IR to total gas cooling. The data in the figure are the observed IRX ratios for the various remnants. The open and solid circles represent the data before and after the observations have been 'corrected' for non-equilibrium ionization effects. The figure shows that for all remnants (with the possible exception of SN 1006 and RCW 103), the IRX ratio is significantly larger than unity. These results clearly demonstrate that IR emission, mainly attributed to gas-grain collisions, is the dominant cooling mechanism in these SNR over large periods of their evolutionary lifetime. The same qualitative conclusion was reached by Graham et al. (1987; see also this volume), who compared the IR and X-ray emission of various SNR in the Large Magellanic Cloud. They found that on the average, remnant cooling by IR emission dominates the atomic cooling by a factor of $\sim 10$.

\section{The Infrared Diagnostic of a Supernova Remnant}

\section{(a) Plasma Parameters}

An important quantity that can be derived from the IR observations of a remnant is the temperature of the radiating dust. It is independent of any assumed distance to the remnant, and can be obtained by a simple fit to the IR spectrum without any major assumptions about the dust model. Figure 7 depicts the temperature, $T_{d}$, of $0.1 \mu \mathrm{m}$ silicate and graphite grains that are collisionally heated by a plasma with an electron density of $1 \mathrm{~cm}^{-3}$, as a function of electron temperature $T$. As in the behavior of $\Lambda_{d}(T)$, the dust temperature initially rises with $T$ as all the energy of the impinging electrons is deposited in the dust. Above a given gas temperature $\left(T \approx 2 \times 10^{7}\right.$ $K)$, the electrons penetrate the grains and $T_{d}$ reaches a plateau value of $\approx 58 \mathrm{~K}$. This behavior of $T_{d}$ versus $T$ suggests that we should, for the purpose of the analysis, divide all remnants into two categories: young remnants, defined here as remnants in which the postshock temperature is $\geq$ $2 \times 10^{7} \mathrm{~K}$ (or expansion velocities above $\sim 1200 \mathrm{~km} \mathrm{~s}^{-1}$ ); and older remnants, characterized by postshock temperatures $\leq 2 \times 10^{7} \mathrm{~K}$ (or expansion velocities below $\sim 1200 \mathrm{~km} \mathrm{~s}^{-1}$ ). In young remnants $T_{d}$ is essentially independent of the gas temperature, whereas in older remnants the dust temperature 'merely' constrains the allowable combinations of plasma densities and temperatures.

In young remnants, dust temperatures above or below $58 \mathrm{~K}$ can only be achieved by respectively increasing or decreasing the gas density from $1 \mathrm{~cm}^{-3}$. As a result, the $I R$ emission from these objects is an excellent diagnostic of plasma density. Figure 8 illustrates this point for the three historical remnants, Kepler, Cas A, and Tycho. The figure depicts the dust temperature (of an $0.1 \mu \mathrm{m}$ graphite grain) as a function of plasma density for two values, $\mathrm{T}=2 \times 10^{7}$ and $10^{8} \mathrm{~K}$, of gas temperature which bracket all possible values in these remnants. The proximity of the curves illustrates the insensitivity of $T_{d}$ on $T$. Dust temperatures in these remnants are (Dwek et al. 1987a; Braun 1987; Arendt 1987b) $\sim 100,85$, and $80 \mathrm{~K}$, respectively. The figure shows that the corresponding plasma densities in these remnants are $\sim 20,10$, and $7 \mathrm{~cm}^{-3}$. Uncertainties in these derived values are $\sim 50 \%$. Even though these results were derived for a specific grain size and composition, a careful analysis shows that in young remnants, the dust temperature is not very dependent on grain size or composition (Dwek 1987). Electron densities derived from X-ray observations are $0.4-4.6 \mathrm{~cm}^{-3}$ for Tycho (detailed explanations for the range of values given here are given by Seward, Gorenstein, and Tucker 1983); $6 \mathrm{~cm}^{-3}$ for Cas A (Murray et al. 1979); and 7 $\mathrm{cm}^{-3}$ for Kepler (Matsui et al. 1984). The agreement between the IR and X-ray derived densities is excellent for Cas A and Kepler, but quite poor for Tycho. This discrepancy should clearly be a subject for further investigation. 


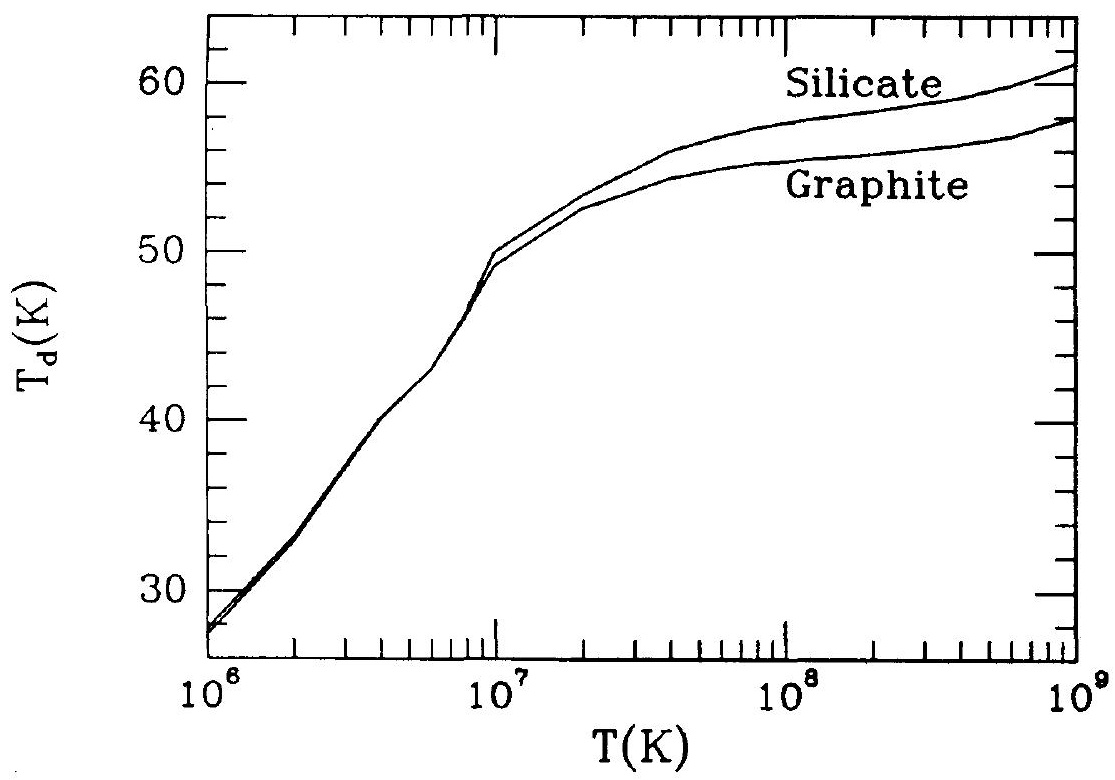

Figure 7 - The temperature of $0.1 \mu \mathrm{m}$ silicate and graphite grains is depicted as a function of gas temperature $T$, for a gas density of $1 \mathrm{~cm}^{-3}$.

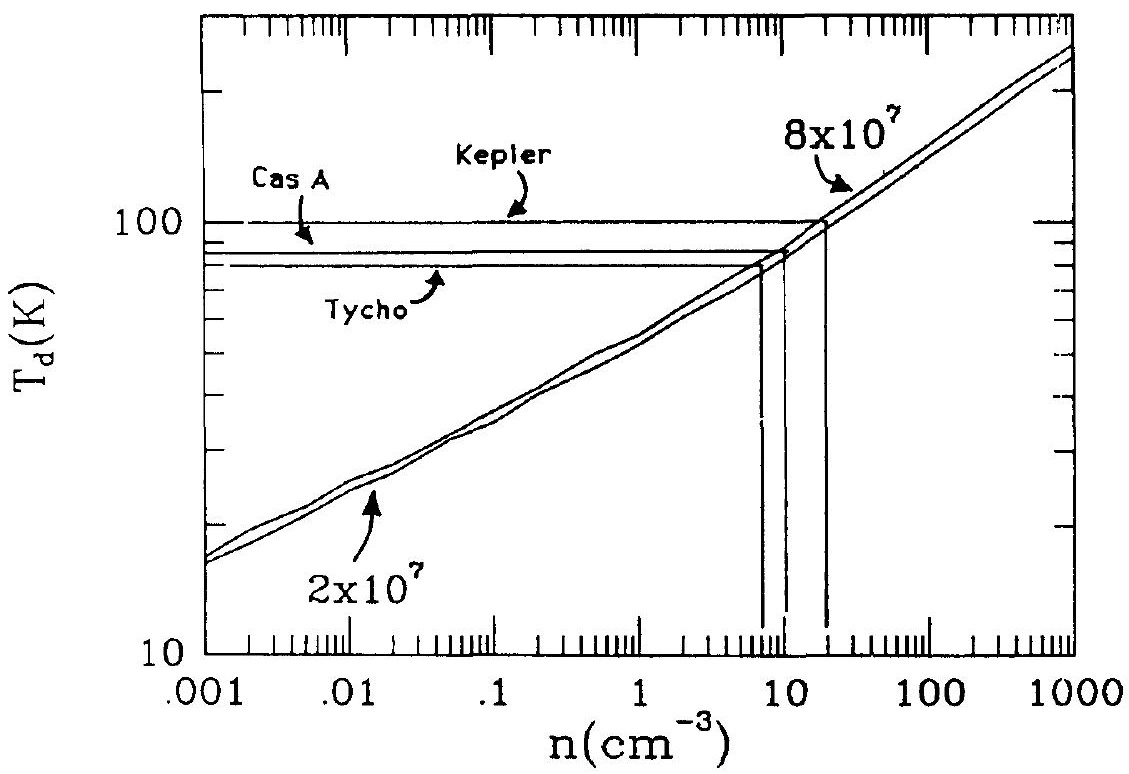

Figure 8 - The temperature of $0.1 \mu \mathrm{m}$ graphite grains is depicted here as a function of gas density $n$, for two values of the gas temperature bracketing the range of values expected in the remnants shown. The figure illustrates how gas densities of young remnants can be derived from their dust temperatures.

For older remnants $T_{d} \sim n^{\alpha} T^{\beta}$, where $(\alpha, \beta)$ are $(0.20,0.30)$ and $(0.17,0.25)$ for $0.1 \mu \mathrm{m}$ graphite and silicate grains, respectively. The above relation is of little use in practical applications 
since, unlike in young remnants, the dust temperature is strongly determined by the grain size. A more useful approach, therefore, is to use the planar shock models of Draine (1981) for the analysis of older remnants. In these models Draine presented the IR flux emitted by an MRN distribution of grain sizes for various gas densities, and shock velocities ranging from 200 to $1000 \mathrm{~km} \mathrm{~s}^{-1}$. The peak of the IR emission (determined by the range of grain temperature behind the shock) is related to the gas density and shock velocity (or plasma temperature) and given by $\lambda_{\text {peak }}(\mu \mathrm{m}) \approx 75$ $\mathrm{n}_{\mathrm{H}}-0.18 \mathrm{v}_{\mathrm{S}}-0.30$, where $\mathrm{n}_{\mathrm{H}}$ is the H-density of the unperturbed ISM, and $\mathrm{v}_{\mathrm{S}}$ is the shock velocity in $1000 \mathrm{~km} \mathrm{~s}^{-1}$. This relation has been used by Braun (1986a, 1986b, 1986c, and 1987) in his analysis of the various remnants. Two new developments took place since the publication of these models: the first was the realization that an interstellar dust component consisting of very small grains is prevalent in the ISM (e.g. Weiland et al. 1986; and references therein); and the second is that these very small dust particles are stochastically heated in a hot gas (Dwek 1986). The wavelength of peak $\mathbb{R}$ emission will therefore shift, with respect to their position in Draine's models, in much the same way it shifted when very small particles were included in models for the IR cirrus clouds (Draine and Anderson 1985; Weiland et al. 1986). Since various remnant parameters are very sensitive to the value of $\lambda_{\text {peak }}$, one must carefully consider the uncertainties in the derived parameters that result from adopting the above relation.

\section{(b) X-ray Emitting Mass}

From the infrared observations one can derive the mass of dust neccesary to account for the IR emission. The dust mass is directly proportional to the IR luminosity, and therefore dependent on dust temperature (to the power of $\sim 5$ to 6 ) and remnant distance. The mass of swept-up X-ray emitting gas can then be determined if $Z_{d}$, the dust-to-gas mass ratio in the shocked gas, is known. If the IR emission is attributed to swept-up interstellar dust (instead of dust in the ejecta of the remnant) then the average undepleted value of $Z_{d}$ in a gas with a cosmic abundance of heavy elements is $\approx 0.0075$. However, the dust present in the postshock gas will be depleted relative to the unperturbed ISM due to sputtering. This depletion factor can be estimated if the density of the postshock gas and the age of the remnant are known. The mass determined from this analysis can be compared to the mass of swept-up interstellar gas. For example, in Cas A the dust mass was found to be $\sim 0.004 \mathrm{M}_{\odot}$ (Dwek et al. 1987a). The postshock depletion factor is $\sim 0.64$, giving a value of $0.8 \mathrm{M}_{\odot}$ for the $\mathrm{X}$-ray emitting gas. From our previous analysis we deduced a postshock density of $\sim 8 \mathrm{~cm}^{-3}$ in Cas $\mathrm{A}$. The radius of the remnant is $1.7 \mathrm{pc}$, giving a value of $1.4 \mathrm{M}_{\odot}$ for the mass of swept-up gas. This value is in reasonable agreement with the previous determination. The difference may, however, imply a filling factor $<1$ for the $2 \mathrm{~cm}^{-3}$-density phase of the medium around the remnant. Braun (1987) deduced a value of $\sim 1.3 \mathrm{M}_{\odot}$ for the shocked ISM mass in Cas A. Similar values were obtained by Braun for Tycho and Kepler. These values compare favorably with the value of $\sim 3.5 \mathrm{M}_{\odot}$ derived by Murray et al. (1979) from the X-ray analysis of Cas A, if non-equilibrium ionization effects are taken into account. For the Cygnus Loop, Dwek (1987) deduced a mass of $\sim 70 \mathrm{M}_{\odot}$, with an uncertainty of about a factor of $\sim 2$. This value is in good agreement with the value of $\sim 100 \mathrm{M}_{\odot}$ derived by $\mathrm{Ku}$ et al. (1984) from the $\mathrm{X}$-ray analysis of the remnant.

\section{(c) Remnant Energetics}

From the dynamics of the remnant and the mass of swept-up interstellar gas, one can infer $\mathrm{E}_{\mathrm{k}} \mathrm{ISM}$, the kinetic energy of the shocked ISM. This quantity can be related to $\mathrm{E}_{\mathrm{o}}$, the energy of the explosion, if the evolutionary state of the remnant is known. For a remnant that has entered the 
adiabatic stage of its evolution, $\mathrm{E}_{\mathrm{k}} \mathrm{ISM}=0.28 \mathrm{E}_{\mathrm{o}}$ (Chevalier 1974). From an analysis of the dynamics of Cas A, Braun (1987) deduced that the kinetic energy of the swept-up ISM is $4.2 \times 10^{49}$ erg, and that the remnant evolved beyond the adiabatic stage of its evolution. These two facts lead then to an initial energy $E_{O}$ of $1.5 \times 10^{50}$ erg. Values of $E_{o}=1.4 \times 10^{50}$ and $1.8 \times 10^{50}$ erg were derived for Kepler and Tycho, respectively. The major uncertainty in these values arises from the uncertainty in the evolutionary stage of the remnant. The analysis of Braun implies that the mass of diffuse ejecta in these remnants is $\sim 0.3-0.4 \mathrm{M}_{\odot}$. This may be problematic for $\mathrm{Cas} A$, which is believed to be the result of the explosion of a massive star, unless most of the material was ejected in dynamically non-interacting clumps.

\section{(d) IR Morphology of SNR}

The medium into which a supernova is expanding is inhomogeneous. The presence of density gradients and clouds in the ambient medium affect the propagation of the supernova blast wave (McKee 1987), and will have an important influence on the IR appearance of a remnant. For example, a comparison of the IR and X-ray emission from RCW 86 shows a very good correlation between the two images in the soutwestern part of the remnant (see Figure 9). However, no IR emission is detected from the $\mathrm{X}$-ray emitting gas in the east. This suggests that the remnant is expanding into a steep east-west density gradient, and that a minimum ISM density is required to detect the $\mathbb{R}$ emission from the shocked gas. The same conclusion was reached by Pisarski,Helfand, and Kahn (1984) from their X-ray analysis of the remnant. Comparison of the IR and X-ray morphology of Cas A (Dwek et al. 1987a) suggests the presence of non-uniform extinction across the remnant. Furthermore, differences between the IR brightness in the northern and southern parts of the remnant suggest the presence of a density gradient between the front and back part of the remnant (Braun 1987). A comparison among the optical, X-ray, and IR emission from the Cygnus Loop (Figure 3) shows enhanced IR emission associated with the optical line emitting filaments encountered by the shock.
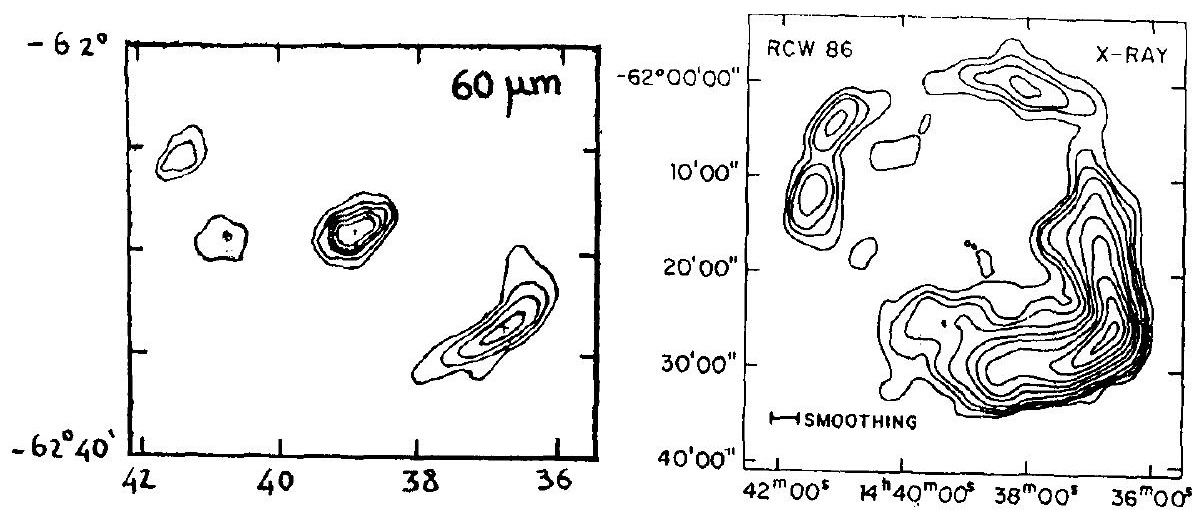

Figure 9 - Comparison of the IR (Figure 9a) and Einstein X-ray image (Figure 6b) of RCW 86. 
An IR analysis of different regions in a remnant can put valuable constraints on the existence of various gas 'phases' and their filling factor in its interior. For example, the IR spectrum of Cas A is very well approximated by a single-temperature dust component with $T_{d} \sim 80-90 \mathrm{~K}$. This fact can be used to constrain the density contrast and the filling factor of the two phases responsible for the double hemisphere structure that was pointed out by Braun. A density contrast of $\sim 10$ will suggest that the dust temperature in the low-density phase is $\sim 50-60 \mathrm{~K}$. Even though the IR luminosity of the dust residing in this phase will be reduced by a factor of $\sim 10$, it may still contribute considerably to the 60 and $100 \mu \mathrm{m} f l u x$ from the remnant, depending on its filling factor. Braun (1987) suggested a filling factor of $\sim 0.9$ for this low-density phase, which may be

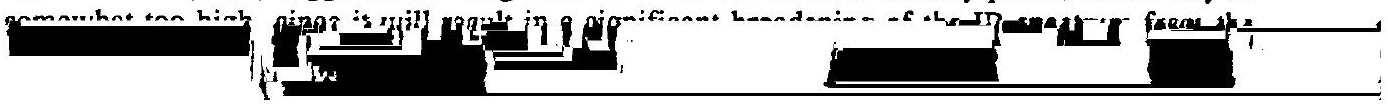

ificantly shorter than the time between electronic collisions, so that the dust cools to a erature, $\mathrm{T}_{\min }$, well below its equilibrium temperature before it is hit by another electron. The It is that the dust temperature fluctuates between $T_{\min }$ and $T_{\max }$, with most of the energy sited in the collision radiated at $T_{\max }$. Figure 11 is a schematic illustration of the effects of erature fluctuations in a very small dust particle that is immersed in a hot gas. The small dust cles needed to explain the excess $12 \mu \mathrm{m}$ emision in Cas A could not have been created by the ion of larger grains in shock - the remnant is too young for this process to be significant. Very 11 grains must therefore be present in the ambient medium into which the remnant is expanding. IRAS observations of Cas A therefore constitute the first observational evidence for the alence of these very small dust grains around SNR, as well as in the general ISM.

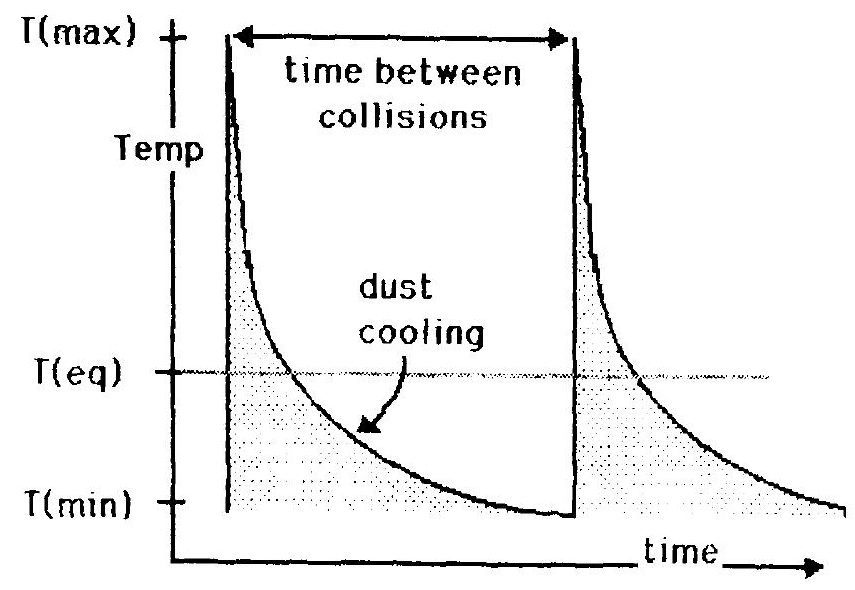

ure 11 - A graphic representation of the temporal behavior of a collisionally-heated, very !ll, dust particle. Following a single electronic collision, the temperature of a $20 \AA$ dust particle, redded in a $10^{7} \mathrm{~K}$ eas. will suree to a value of $\sim 600 \mathrm{~K}$. The eauilibrium value of the dust 


\section{Effect on Remnant Evolution}

An important question is - will the IR cooling of the remnants accelerate their evolution compared with the standard evolutionary picture presented, for example, by Cox (1972)? The first evolutionary stage of the remnant is the free expansion phase, terminated when enough mass has been swept up to begin decelerating the shock. This stage is not affected by radiative processes behind the shock. The second stage of remnant evolution, the adiabatic phase, is terminated when about half of the thermal energy of the remnant has been radiated away. The end of this phase is marked by the formation of a dense shell behind the shock and the beginning of the "snowplow" phase of remnant evolution. Enhanced cooling resulting from gas-grain collisions may therefore significantly affect the duration of the adiabatic phase.

The importance of dust cooling depends on the density of the ISM, and can be qualitatively assessed in the following way: Consider a remnant with $E_{0}=10^{51}$ erg expanding into an ISM with $n_{0}=1 \mathrm{~cm}^{-3}$. The postshock temperature at the end of the adiabatic phase, $T_{c}$, is about $5 \times 10^{5} \mathrm{~K}$, whereas IR emission dominates the cooling of the remnant when $T>6 \times 10^{5} \mathrm{~K}$ (see Figure 4). Gas-grain collisions will therefore have only a moderate effect on the duration of the adiabatic phase, since most of the thermal energy is lost from the remnant when its volume is largest (i.e. around temperatures of $\sim 6 \times 10^{5} \mathrm{~K}$ when IR and atomic cooling are about equally important). At densities of $n_{0}=100 \mathrm{~cm}^{-3}, T_{c}$ is about $2 \times 10^{6} \mathrm{~K}$, so that most of the thermal energy is lost when IR emission dominates the cooling by a large factor. Calculations by Dwek (1981) show that the adiabatic phase is shortened by only $\sim 10 \%$ in the former case, and by as much as $\sim 40 \%$ in the latter case. At higher densities, IR cooling should be more pronounced. However, grain destruction becomes increasingly important as well, resulting in declining effect of gas-grain collisions on remnant evolution. All adiabatic remnants considered here expand into media with densities $<10$ $\mathrm{cm}^{-3}$, so that the IR emission will have a negligible effect on their general evolution. However, as suggested by Graham et al. (1987), the IR emission will affect the internal temperature structure of the remnant.

\section{Future Prospects}

The IRAS all sky survey opened a new wavelength window for the studies of SNR. An interesting question is whether SNR form a class of IR objects that is distinct from other known IR sources like HII regions or planetary nebulae. Such a distinct spectral 'signature' will prove valuable in the identification of SNR that may explode in molecular clouds (Shull 1980; Wheeler, Mazurek, and Sivaramakrishnan 1980). The preliminary investigation of Arendt (1987a) shows that the IR spectrum of an 'average' SNR peaks between the 60 and $100 \mu \mathrm{m}$ bands of the IRAS, or that it is still rising at $100 \mu \mathrm{m}$. The spectrum of these remnants is therefore essentially identical to that of compact HII regions. Planetary Nebulae are hotter objects with IR spectra that peak between 25 and $60 \mu \mathrm{m}$ (e.g. Pottash 1984). They are therefore distinctly different from the average SNR but look very similar to young remnants like Tycho, Kepler, and Cas A. The lack of any unique IR spectral signature and the confusion with IR sources near the galactic plane suggest that the most promising approach to discover new SNR is still to search for their radio emission (Caswell 1987).

However, even if the IR cannot currently be used to identify new remnants, it provides researchers with powerful means to study the dynamics and energetics of existing remnants, and their interaction with the ambient interstellar medium. A stepwise approach to such analysis would include the following: removal of zodiacal, galactic, and cirrus emission components from the IR image of the remnant; analysis of the relative contribution of IR lines and continuum radiation to the emission; comparison between the IR and X-ray emission to estimate the contribution of collisionally-heated dust to the emission; comparison between IR and optical, HI, and CO maps of 
the region to estimate the contribution of IR lines from shock-heated filaments or clouds; search for adjacent $\mathrm{OB}$ associations to estimate the relative contribution of radiatively-heated dust to the emission. This outline shows the intricate relation between studies at the various wavelengths at our disposal, a collection of which IR radiation is the newest member.

\section{Acknowledgements}

I thank Robert Braun for helpful conversations. Walter Rice provided the infrared images presented in the figures. Ted Gull provided the optical image of the Cygnus Loop digitized by Dan Klinglesmith, III. Fred Seward provided the X-ray image of the Cygnus Loop, and Ryszard Pisarski provided that of RCW 86.

\section{References}

Arendt, R. G. 1987a, this volume.

Arendt, R. G. 1987 b, private communications.

Braun, R. 1986a, Astr. Ap, 162, 259.

. 1986b, Astr. Ap, 164, 193.

1986c, Astr. Ap., 164, 208.

1987, Astr. Ap, 171, 233.

Burke, J. R., and Silk, J. 1974, Ap. J., 190, 1.

Caswell, J. L. 1987, this volume.

Chevalier, R. A. 1974, Ap.J, 188, 501.

Cox, D. P. 1972, Ap. J, 178, 159.

Dinerstein, H. L., Lester, D. F., Rank, D. M., Werner, M. W., and Wooden, D. H. 1987, Ap. J, 312, 314.

Draine, B. T. 1981, Ap. J, 245, 880.

Draine, B. T., and Salpeter, E. E. 1979a, Ap. J, $231,77$.

Draine, B. T., and Salpeter, E. E. 1979b, Ap. J, 231, 438.

Draine, B. T., and Anderson, N. 1985, AD.J., 292, 494.

Dwek, E. 1981, Ap. J, 247, 614.

Dwek, E., and Werner, M. W. 1981, Ap. J, 248, 138.

Dwek, E. 1986, Ap. J, 302, 363.

Dwek, E., Dinerstein, H. L., Gillett, F. C., Hauser, M. G., and Rice, W. L. 1987a, Ap. J, 315, 571.

Dwek, E. 1987, $\underline{A D} . \mathrm{J}$, in press.

Dwek, E., Petre, R., Szymkowiak, A., and Rice, W. L. 1987b, Ap. J (Letters), in press.

Graham, J. R., Evans, A., Albinson, J. S., Bode, M. F., and Meikle, W. P. S. 1987, Ap. J, 319, 000.

Hamilton, A. J. S., Sarazin, C. L., and Chevalier, R. A. 1983, Ap. J. Suppl., 51, 115 (HSC).

Harvey, P. M., Gatley, I., and Thronson, H. A. 1978, Pub. A. S. P., 90, 143.

Hauser, M. G., et al. 1984, Ap. J. (letters), 278, L15.

Ku, W. H.-M., Kahn, S. M., Pisarski, R. L., and Long, K. S. 1984, Ap. J, 278, 615 .

Low, F. J., et al. 1984, Ap. J. (letters), 278, L19.

Marsden, P. L., Gillett, F. C., Jennings, R. E., Emerson, J. P., de Jong, T., Olnon, F. M. 1984, Ap. J. (Letters), 278, L29.

Mathis, J. S., Rumpl, W., and Nordsieck, K. H. 1977, Ap. J, 217, 425.

Matsui, Y., Long, K. S., Dickel, J. R., and Greisen, E. W. 1984, Ap. J., 287, 295.

McCollough, M. L., and Mufson, S. L. 1987, this volume. 
McKee, C. F. 1987, this volume.

Mezger, P. G., Tuffs, R. J., Chini, R., Kreysa, E., and Gemund, H.-P. 1986, Astr. Ap., 167, 145.

Mufson, S. L., McCollough, M. L., Dickel, J. R., Petre, R., White, R., and Chevalier, R. 1986, A. J. $92,1349$.

Murray, S. S., Fabbiano, G., Fabian, A. C., Epstein, A., and Giacconi, R. 1979, Ap. J. (Letters), 234, L69.

Neugebauer, G. et al. 1984, Ap. J. (Letters), 278, L1.

Ostriker, J. P., and Silk, J. 1973, AD. J. (Letters), 184, L1 13.

Pisarski, R. L., Helfand, D. J., and Kahn, S. M. 1984, Ap. J, 277, 710.

Pottash, S. R. 1984, Planetary Nebulae (Dordrecht: Reidel), page 302.

Raymond, J. C., Cox, D. P., and Smith, B. W. 1976, Ap. J, 204, 290.

Seab, C. G. 1986, Interstellar Processes, eds. D. Hollenbach, and H. A. Thronson, Jr. (Dordrecht: Reidel).

Seward, F., Gorenstein, P., and Tucker, W. 1983, Ap. J., 266, 287.

Shull, J. M. 1977, Ap. J., 215, 805.

Shull, J. M. 1980, Ap. J, 237, 769.

Silk, J., and Burke, J. R. 1974, Ap. J., 190, 11.

Spitzer, L. 1978, Physical Processes in the Interstellar Medium (New York: Interscience), p. 199.

Weiland, J. L., Blitz, L., Dwek, E., Hauser, M. G., Magnani, L., and Rickard, J. L 1986, Ap. J. (Letters), 306, L101.

Wheeler, J. C., Mazurek, T. J., and Sivaramakrishnan, A. 1980, Ap. J., 237, 781.

Wright, E. L., Harper, D. A., Lowenstein, R. F., Keene, J., and Whitcomb, S. E. 1980, Ap. J. (Letters), 240, L157. 
McKee, C. F. 1987, this volume.

Mezger, P. G., Tuffs, R. J., Chini, R., Kreysa, E., and Gemund, H.-P. 1986, Astr. Ap., 167, 145.

Mufson, S. L., McCollough, M. L., Dickel, J. R., Petre, R., White, R., and Chevalier, R. 1986, A. J. $92,1349$.

Murray, S. S., Fabbiano, G., Fabian, A. C., Epstein, A., and Giacconi, R. 1979, Ap. J. (Letters), 234, L69.

Neugebauer, G. et al. 1984, Ap. J. (Letters), 278, L1.

Ostriker, J. P., and Silk, J. 1973, AD. J. (Letters), 184, L1 13.

Pisarski, R. L., Helfand, D. J., and Kahn, S. M. 1984, Ap. J, 277, 710.

Pottash, S. R. 1984, Planetary Nebulae (Dordrecht: Reidel), page 302.

Raymond, J. C., Cox, D. P., and Smith, B. W. 1976, Ap. J, 204, 290.

Seab, C. G. 1986, Interstellar Processes, eds. D. Hollenbach, and H. A. Thronson, Jr. (Dordrecht: Reidel).

Seward, F., Gorenstein, P., and Tucker, W. 1983, Ap. J., 266, 287.

Shull, J. M. 1977, Ap. J., 215, 805.

Shull, J. M. 1980, Ap. J, 237, 769.

Silk, J., and Burke, J. R. 1974, Ap. J., 190, 11.

Spitzer, L. 1978, Physical Processes in the Interstellar Medium (New York: Interscience), p. 199.

Weiland, J. L., Blitz, L., Dwek, E., Hauser, M. G., Magnani, L., and Rickard, J. L 1986, Ap. J. (Letters), 306, L101.

Wheeler, J. C., Mazurek, T. J., and Sivaramakrishnan, A. 1980, Ap. J., 237, 781.

Wright, E. L., Harper, D. A., Lowenstein, R. F., Keene, J., and Whitcomb, S. E. 1980, Ap. J. (Letters), 240, L157. 